\title{
Devriesea agamarum associated cheilitis in a North African spiny-tailed lizard (Uromastyx acanthinura) in Spain
}

\author{
Miguel Gallego ${ }^{1, *}$, Carles Juan-Sallés ${ }^{2}$ and Tom Hellebuyck ${ }^{3}$ \\ ${ }^{1}$ Centro Veterinario Madrid Exóticos, Meléndez Valdés 17, 28015 Madrid, Spain \\ ${ }^{2}$ Noah's Path, Arquitecto Santiago Pérez, Aracil 30 bajo, 03203 Elche, Spain \\ ${ }^{3}$ Department of Pathology, Bacteriology and Avian Diseases, Faculty of Veterinary Medicine, Ghent University, \\ Salisburylaan 133, B-9820 Merelbeke, Belgium
}

\begin{abstract}
An 11-year-old, male North African spiny-tailed lizard (Uromastyx acanthinura) was presented with hyperkeratotic cheilitis. Based on clinical examination, histology and microbiological testing, Devriesea agamarum was identified as the causative agent of the dermal disorder. Moreover, an abundant culture of $D$. agamarum was obtained following sampling of the oral cavity. Cheilitis was present for three years in the spiny-tailed lizard and during the first year of that period the lizard was housed together with an ocellated lizard (Timon lepidus), an Algerian orange-tailed skink (Eumeces algeriensis) and a female $U$. acanthinura. The latter lizard showed signs of chronic dermatitis and had deceased approximately 2.5 years prior to initial presentation of the male dab lizard because of renal failure. The other cohabiting lizards showed no dermal lesions and D. agamarum could not be demonstrated following dermal, cloacal and oral sampling. Recurrence of the bacterial skin infection was observed following a first antimicrobial treatment. This was considered to result from failure to eliminate the bacterium from the treated dab lizard or re-infection from the environment or asymptomatic carriers. A second treatment, including disinfection of the enclosure with household bleach $0.1 \%$, weekly mechanical debridement of the crusty lesion and treatment with ceftazidime at $20 \mathrm{mg} / \mathrm{kg}$ intramuscularly every 72 hours for 57 days resulted in resolution of the skin lesions, elimination of D. agamarum from the oral cavity based on repetitive microbiological sampling and no recurrence of the lesions during a 31 month follow up period. The present case is the first report of devrieseasis in Spain and highlights the importance of a multidirectional diagnostic and therapeutic approach towards controlling devrieseasis in captive lizard collections. Several disease aspects such as persistency are discussed in the light of the contemporary available literature.
\end{abstract}

Keywords: Cheilitis, Dermatitis, Devriesea agamarum, Uromastyx acanthinura.

\section{Introduction}

Devriesea agamarum has been isolated previously in captive lizards from Belgium, Austria, Switzerland, Croatia and from free-ranging lizards species from the French Caribbean island of Saint Barthélemy (Martel et al., 2008; Lukac et al., 2013; Schmidt-Ukaj et al., 2014; Rossier et al., 2016; Hellebuyck et al., 2017).

The bacterium is considered worldwide as an important agent of dermal disease and/or septicaemia that has eradicated entire captive collections of lizards. Moreover, the bacterium was recently demonstrated to affect free-ranging endangered Antillean iguanas (Iguana delicatissima) and should be considered as one of the first bacterial diseases that might pose a conservation threat to free-ranging squamates (Hellebuyck et al., 2017).

This is the first reported case of devrieseasis in Spain; moreover, several disease aspects such as persistency are discussed in the light of the contemporary available literature.

\section{Case Details}

An 11-year-old male North African spiny-tailed lizard (Uromastyx acanthinura) weighing $800 \mathrm{~g}$, purchased at a reptile pet shop in Madrid eight years before initial presentation, was presented with a history of progressive crusty cheilitis since three years. Since two months, a $10 \%$ povidone-iodine solution was topically applied on a daily basis by the owner and resulted in a mild improvement of the skin lesions. The spiny-tailed lizard was housed with a female during a one-yearperiod that died 2.5 years prior to initial presentation of the male at the age of 24 years. The female had been raised in captivity since birth and was born out of wild caught animals originating from Morocco. Besides showing cheilitis and dermatitis with an identical clinical appearance as observed in the male, severe renal and visceral gout were determined as the eventual cause of death in the female spiny-tailed lizard based on gross pathological and histopathological examination. 
An adult male Algerian orange-tailed skink (Eumeces algeriensis) and a female ocellated lizard (Timon lepidus) of unknown age were introduced to the same enclosure as the male Uromastyx a couple of months after the female had died but remained clinically unaffected.

At the time of initial presentation, four full thickness biopsies were collected from the affected area of skin in the male lizard for microbiological and histopathological examination. Anesthesia was performed with intravenous propofol into the ventral tail vein (Lipuro®, B. Braun Spain, 08191 Barcelona, Spain; $10 \mathrm{mg} / \mathrm{kg}$ ). The biopsies were fixed in formalin and embedded in paraffin followed by hematoxylin and eosin (HE) staining as well as gram staining. Histological evaluation of the sections of the skin biopsies revealed severe multifocal epidermal hyperplasia with marked hyperkeratosis, mild multifocal infiltration of lymphocytes and heterophils in the dermis with heterophilic exocytosis, intercellular edema and intralesional, predominantly filamentous gram positive bacteria with a beaded appearance and occasional branching within the keratin layers. Abundant growth of a gram negative bacillus belonging to the Enterobacteriaceae that could not be identified using conventional biochemical testing was observed following culturing of the skin lesion. The latter isolate was considered as a contaminant or normal part of the skin microbiota taking into account the presence of exclusively gram positive beaded and branching filamentous bacteria in the histological sections of the skin biopsies.

Treatment with ceftazidime (Ceftazidima Normon inyectable 1g. Laboratorios Normon, 28760 Madrid, Spain; $15 \mathrm{mg} / \mathrm{kg}$ intramuscularly every 72 hours) was initiated during three weeks and resulted in complete resolution of the skin lesions. During the next months, however, cheilitis gradually reappeared and the severity of the skin lesions was identical to those observed during initial presentation approximately eighteen months later (Figure 1).

At that moment, swabs were collected from the oral cavity and the scaly lip lesions as well as crusts that were obtained following debridement of the crusty lesions for microbiological examination. All samples were cultured during $24-48 \mathrm{~h}$ on colistin nalidixic acid (CNA, Oxoid GmbH, Wesel, Germany) agar at $37^{\circ} \mathrm{C}$ and $5 \% \mathrm{CO}_{2}$. Pure cultures and abundant growth of small, whitish colonies of gram positive bacteria with a narrow zone of hemolysis were observed following culturing of the skin and oral samples respectively. Based on morphological aspects and the results of API Coryne, API 20 STREP, API $50 \mathrm{CH}$ (bioMérieux, Marcy l'etoile, France) testing, the isolates were identified as D. agamarum.

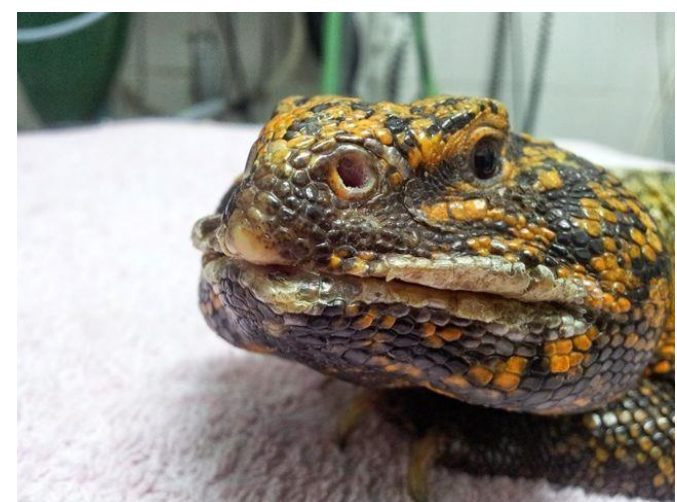

Fig. 1. Crusting lesions in lips of an Uromastyx acanthinura with devrieseasis.

Following 16S rRNA gene sequencing as previously described (Martel et al., 2008), $100 \%$ similarity with the type strain of $D$. agamarum $\left(\mathrm{IMP} 2=\mathrm{LMG} 24257^{\mathrm{T}}\right.$ ) was revealed for all isolates.

In addition, a radiological study of the head and whole body, as well as ultrasound were performed in the spiny-tailed lizard but did not reveal bone lesions, masses or other abnormalities, that could indicate disseminated bacterial infection.

Blood was collected by venipuncture of the ventral coccygeal vein in a heparinized tube for a serum biochemistry profile and hematologic evaluation at the time of radiological examination. A microcapillar filled with heparinized blood was centrifuged at $15,800 \mathrm{rpm}$ for 2 minutes (StatSpin ${ }^{\circledR}$ VT, Iris Sample Processing, Massachusetts 02090-1825, USA) for hematocrit determination. The total white blood cell count by the Natt and Herrick method was obtained with heparinized blood (Campbell and Ellis, 2007).

The rest of the blood was centrifuged at $15,800 \mathrm{rpm}$ for 90 seconds in the same centrifuge and the plasma obtained was used to obtain a basic biochemical profile (Bird/Reptile Rotor Profile for VetScan ${ }^{\circledR}$ VS2, Abaxis, California 94587, USA). Blood biochemistry and hematology results and reference values are presented in Table 1. To date, reference values for North African spiny-tailed lizards are not available in the literature, so values from closely related species (Uromastyx aegyptia microlepis and Uromastyx leptieni) were used for guidance (Naldo et al., 2009). Uric acid, albumin and aspartate aminotransferase were mildly raised (Table 1). Dehydration and mild tissue damage due to loss of appetite and chronic dermal inflammation were considered to most likely have caused these biochemical alterations (Campbell, 2014). Samples from the oral cavity and skin obtained from the orangetailed skink and the ocellated lizard that cohabited with the dab lizard were submitted to microbiological examination as described above but cultured negative for D. agamarum. 
Table 1. Hematology and blood chemistry of a North African spiny-tailed lizard (Uromastyx acanthinura) with devrieseasis.

\begin{tabular}{|c|c|c|}
\hline Parameter & $\begin{array}{c}\text { Uromastyx } \\
\text { acanthinura } \\
\text { with } \\
\text { devrieseasis } \\
\end{array}$ & $\begin{array}{c}\text { Reference values } \\
(U . \text { aegyptia } \\
\text { microlepis and } U . \\
\text { leptieni })^{*}\end{array}$ \\
\hline $\operatorname{PCV}(\%)$ & 31 & $4.9-44.5$ \\
\hline WBC & 5.2 & $1-8.1$ \\
\hline Heterophils (x1000) & 3.64 & $0.59-5.36$ \\
\hline Lymphocytes (x1000) & 1.3 & $0.27-4.05$ \\
\hline Monocytes (x1000) & 0.26 & $0-0.5$ \\
\hline Total protein $(\mathrm{g} / \mathrm{dL})$ & 5.2 & $2.6-7.4$ \\
\hline Albumin (g/dL) & $3.3^{* *}$ & $1.2-3.1$ \\
\hline Globulins (g/dL) & 1.9 & - \\
\hline Uric acid (mg/dL) & $8.78^{* *}$ & $0.3-7.3$ \\
\hline Glucose (mg/dL) & 188 & $67.7-355.6$ \\
\hline Calcium (mg/dL) & 11.8 & $7.2-13.2$ \\
\hline Phosphorus (mg/dL) & 4.8 & - \\
\hline Sodium (mEq/L) & 161 & - \\
\hline Potassium $(\mathrm{mEq} / \mathrm{L})$ & 4.1 & - \\
\hline $\mathrm{AST}(\mathrm{UI} / \mathrm{L})$ & $183^{* *}$ & $28.5-172$ \\
\hline Bile Acids (umol/L) & 37 & - \\
\hline
\end{tabular}

The owner was advised to perform thorough cleaning and disinfection of the terrarium with bleach $0.1 \%$ and ceftazidime was administered at $20 \mathrm{mg} / \mathrm{kg}$ intramuscularly every 72 hours for 57 days. In addition, the crusty skin lesion was debrided mechanically every 7 days until the affected skin site regained its normal appearance. Cheilitis resolved after 50 days of antimicrobial treatment, local debridement and the passing of two shedding cycles (Figure 2). The previously affected area of skin as well as the oral cavity were resampled for microbiological evaluation and tested negative for the presence of D. agamarum.

\section{Discussion}

Currently, Devriesea agamarum is the only member of a newly described genus within the class Actinobacteria. This bacterium is a non-motile, nonspore-forming and non-acid-fast gram positive small rod that occur singly, in pairs or in short chains and that grew on sheep blood agar as small hemolytic mucoid and whitish colonies after $24 \mathrm{~h}$ of incubation at $25-42^{\circ}$ $\mathrm{C}$ under aerobic, microaerophilic or anaerobic conditions (Martel et al., 2008). Partial ribosomal RNA gene sequences and the full genome sequence of the $D$. agamarum strain IMP2 and a partial $16 \mathrm{~S}$ ribosomal RNA gene sequence from a bearded dragon with concurrent Chrysosporium guarroi infection have been deposited in the European Nucleotide Archive (Schmidt-Ukaj et al., 2014; Haesendonck et al., 2015).

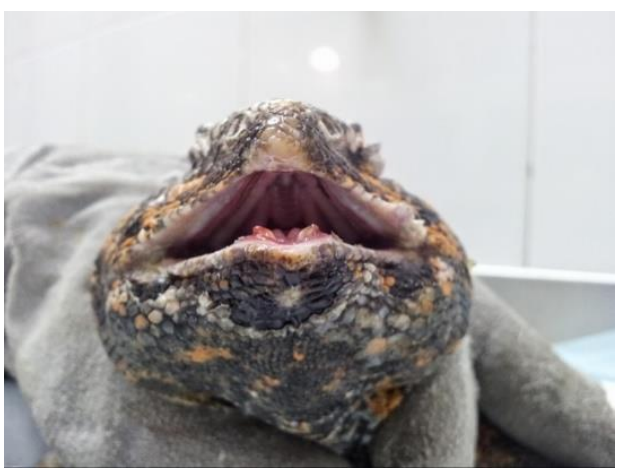

Fig. 2. Lips in shedding after ceftazidime $20 \mathrm{mg} / \mathrm{kg}$ every 48 hours for 57 days.

While disease associated with this bacterium initially seemed restricted to dry land lizards, recent reports have documented devrieseasis in several lizard species that originate from (sub)tropical regions (Bauwens et al., 2014; Rossier et al., 2016; Hellebuyck et al., 2017). Various lizard species can act as asymptomatic carriers in which D. agamarum may be part of the microbiota of the oral cavity or the cloaca (Hellebuyck et al., 2009a; Devloo et al., 2011; Bauwens et al., 2014; Rossier et al., 2016).

The presence of asymptomatic carriers, considered as one of the factors that promote persistency in captive lizard collections, has also been put forward as a possible origin of devrieseasis in free-ranging iguanas (Hellebuyck et al., 2017). Besides the presence of asymptomatical carriers, long term environmental survival has been demonstrated to be another major contributing factor that leads to persistency of devrieseasis (Hellebuyck et al., 2010).

It is possible that persistency of the bacterium in the enclosure of the dab lizard from the present report combined with the presence of D. agamarum in the oral cavity, might explain therapeutic failure following the initial antimicrobial treatment of the dab lizard and recurrence of the skin lesions (Pasmans et al., 2008; Devloo et al., 2011).

Although D. agamarum could not be isolated from the healthy ocellated lizard and the Algerian orange-tailed skink that cohabited with the North African spiny-tailed lizard, screening of healthy lizard should preferably be based on repetitive sampling of oral skin and cloacal swabs as $D$. agamarum is a relatively slow growing coryneform bacterium. Consequently, cultures derived from samples obtained from skin lesions or oral cavities collected in lizards with dermatitis are easily overgrown by other bacteria that might be part of the saurian microbiota.

Unless pure D. agamarum cultures are obtained, the diagnosis of devrieseasis as a cause of dermatological disease in lizards may easily be missed, especially if analyses are performed by microbiological laboratories that are not familiar with the bacterium. 
Following morphological identification, 16S rRNA sequencing is necessary to confirm the identity of the obtained isolate as D. agamarum (Martel et al., 2008). Skin disease in lizards and in other reptiles is primarily the result of inappropriate management in captivity, leading to secondary infection (Hellebuyck et al., 2012). A few pathogens such as D. agamarum, however, may act as primary etiological agents. In the case of $D$. agamarum, a breach of skin integrity seems necessary to allow the bacterium to establish experimental clinical infection (Hellebuyck et al., 2009a; Hellebuyck et al., 2017).

In naturally $D$. agamarum infected lizards, infection also seems to be correlated with traumatic lesions resulting from territorial or reproductive behavior (Bauwens et al., 2014; Hellebuyck et al., 2017). The deceased female spiny-tailed lizard that showed identical skin lesions and was housed together with the male during a one-year-period is presumed as the most likely source of infection. Although infection may have occurred through the environment following contamination by the female lizard, direct contact or infection of biting lesions that are often witnessed during territorial or reproductive behavior in dab lizards, may also have promoted transmission and the onset of clinical infection in the male dab lizard. Indirect factors that impaired skin integrity in the affected male Uromastyx could not be identified.

The most characteristic clinical finding presented in lizards suffering from D. agamarum associated disease is the chronic, crusted dermatitis. Some species, especially Uromastyx spp. and Crotaphytus collaris, show chronic skin lesions associated with high morbidity and low mortality. If the infection persists for a long time, however, the dermal lesions may interfere with normal feeding and foraging behavior and eventually become fatal (Martel et al., 2008; Hellebuyck et al., 2009a; Devloo et al. 2011; Lukac et al., 2013; Bauwens et al., 2014).

In other species such as Agama imperialis, Sauromalus obesus and Physignathus cocincinus, however, high morbidity and mortality is frequently observed resulting from the development of septicemia after showing minimal skin disease in most of the cases (Martel et al., 2008; Pasmans et al., 2008; Rossier et al., 2016).

Devriesea agamarum infection in the male spiny-tailed lizard presented in this case report was limited to the skin and no radiological, hematological or biochemical abnormalities indicative for systemic infection could be demonstrated. Other agents that might cause identical skin disease as seen for $D$. agamarum should be properly excluded (Hellebuyck et al., 2012; SchmidtUkaj et al., 2014).

Especially, dermatophytosis caused by Nannizziopsis spp. and Paranannizziopsis spp. (formerly known as
CANV) in lizards should be differentiated as the clinical presentation as well as the predilection sites of affected skin are highly similar (Paré and Sigler, 2016). In the present case a tentative diagnosis of $D$. agamarum associated cheilitis was based on clinical examination taking into account the species predisposition, but the definitive diagnosis was made by bacteriological examination of the skin lesions and $16 \mathrm{~S}$ rRNA sequencing of the obtained isolate.

Initial treatment with ceftazidime at $15 \mathrm{mg} / \mathrm{kg}$ intramuscularly every 72 hours was sufficient to resolve the skin lesions in the present case, but cheilitis recurred. Accordingly, it was decided to increase the dose of ceftazidime to $20 \mathrm{mg} / \mathrm{kg}$ every 72 hours, clean the enclosure with bleach and debride the lesions once a week. After 57 days of antimicrobial treatment, the resolution of skin lesions and negative cultures as well as the non-recurrence of lesions during a follow-up period of 31 months confirmed the efficacy of the applied treatment and the importance of a multidirectional approach towards the control of $D$. agamarum associated disease. In a reported case series, D. agamarum infected dab lizards that were treated for 15 days with ceftazidime at $10 \mathrm{mg} / \mathrm{kg}$ intramuscularly every 72 hours in conjunction with rinsing the lesions with an antiseptic and surgical debridement in one of the cases resulted in successful outcome in all treated lizards (Lukac et al., 2013).

For the present case, the empirical treatment was presumed to be ineffective due to inadequate circulating levels of the used antimicrobial or insufficient penetration of the poorly vascularized affected skin sites. Because in turtles and snakes the administration of ceftazidime at a dose of 20-22 mg / $\mathrm{kg}$ every 72 hours proved to achieve therapeutic plasma concentrations (Gibbons, 2014), the dose used in the second treatment applied in the Uromastyx in this case report was increased to $20 \mathrm{mg} / \mathrm{kg}$ every 72 hours. Unfortunately, there are no pharmacokinetic / pharmacodynamic studies in relation to the use of ceftazidime in Uromastyx. In the present report, a case of cheilitis caused by Devriesea agamarum is described for the first time in Spain. Based on the available literature and the widespread occurrence of asymptomatic carriers, devrieseasis may be severely underdiagnosed. Mainly difficulties to correctly sample and interpret cultures derived from D. agamarum associated skin lesions may largely contribute to this. The recurrence of the dermal lesions in the present case is a typical feature that is associated with devrieseasis in captive lizard collections and besides the use of appropriate treatment protocols, a multidirectional control strategy is mandatory to resolve environmental survival of the bacterium and to identify asymptomatical carriers, two major factors that promote persistency of the disease. 
Natural or acquired resistance against antimicrobial agents has not been documented so far (Hellebuyck et al., 2009b, 2010), but the importance of susceptibility testing of any D. agamarum isolate cannot be overly emphasized.

\section{Conflict of interest}

The Authors declare that there is no conflict of interest.

\section{References}

Bauwens, L., Vercammen, F., Hendrickx, F., Pasmans, F. and Martel, A. 2014. Prevalence of Devriesea agamarum in the lizard collection of The Royal Zoological Society of Antwerp. J. Zoo Aquar. Res. 2(3), 88-91.

Campbell, T.W. and Ellis, C.K. 2007. Avian and Exotic Animal Hematology and Cytology. $3^{\text {rd }}$ ed. Ames (IA): Blackwell Publishing.

Campbell, T.W. 2014. Clinical pathology. In: Current therapy in reptile medicine and surgery, Eds., Mader, D.R. and Divers, S.J. $1^{\text {st }}$ ed. St. Louis, MO: Saunders, pp: 70-92.

Devloo, R., Martel, A., Hellebuyck, T., Vranckx, K., Haesenbrouck, F. and Pasmans, F. 2011. Bearded dragons (Pogona vitticeps) asymptomatically infected with Devriesea agamarum are a source of persistent clinical infection in captive colonies of dab lizards (Uromastyx sp.). Vet. J. 150, 297-301.

Gibbons, 2014. P.M. Therapeutics. In: Current therapy in reptile medicine and surgery, Eds., Mader, D.R. and Divers, S.J. 1st ed. St. Louis, MO: Saunders, pp: 57-69.

Haesendonck, R., Van Nieuwerburgh, F., Haesenbrouck, F., Deforce, D., Pasmans, F. and Martel, A. 2015. Genome sequence of Devriesea agamarum, isolated from Agamid lizards with dermatitis. Genome Announc. 3(4), e00949-15. doi: 10.1128/genomeA.00949-15.

Hellebuyck, T., Martel, A., Chiers, K., Haesenbrouck, F. and Pasmans, F. 2009a. Devriesea agamarum causes dermatitis in bearded dragons (Pogona vitticeps). Vet. Microbiol. 134(3-4), 267-271.

Hellebuyck, T., Pasmans, F., Blooi, M., Haesenbrouck, F. and Martel, A. 2010. Prolonged environmental resistence requires efficient disinfection procedures to control Devriesea agamarum-associated disease in lizards. Lett. Appl. Microbiol. 52, 28-32.

Hellebuyck, T., Pasmans, F., Haesenbrouck, F. and Martel, A. 2009b. Designing a successful antimicrobial treatment against Devriesea agamarum in lizards. Vet. J. 139(1-2), 189-192.
Hellebuyck, T., Pasmans, F., Haesenbrouck, F. and Martel, A. 2012. Dermatological diseases in lizards. Vet. J. 193(1), 38-45.

Hellebuyck, T., Van Steendam, K., Deforce, D., Blooi, M., Van Nieuwerburgh, F., Bullaert, E., Ducatelle, E., Haesenbrouck, F., Pasmans, F. and Martel, A. 2014. Autovaccination confers protection against Devriesea agamarum associated septicemia but not dermatitis in bearded dragons (Pogona vitticeps). PLoS One. 9(12), e113084. doi: 10.1371/journal.pone.0113084.

Hellebuyck, T., Questel, K., Pasmans, F., Brantegem, L.V., Philip, P. and Martel, A. 2017. A virulent clone of Devriesea agamarum affects endangered Lesser Antillean iguanas (Iguana delicatissima). Sci. Rep. 7, 12491. doi: 10.1038/s41598-01711874-x.

Lukac, M., Horvatec-Tomic, D. and Prukner-Radovcic, E. 2013. Findings of Devriesea agamarum associated infections in spiny-tailed lizards (Uromastyx sp.) in Croatia. J. Zoo Wildl. Med. 44(2), 430-434.

Martel, A., Pasmans, F., Hellebuyck, T., Haesenbrouck, F. and Vandamme, P. 2008. Devriesea agamarum gen. nov., sp. nov., a novel actinobacterium associated with dermatitis and septicaemia in agamid lizards. Int. J. Syst. Evol. Microbiol. 58(9), 2206-2209.

Naldo, J.L., Libanan, N.L. and Samour, J.H. 2009. Health assessment of a spiny-tailed lizard (Uromastyx spp.) population in Abu Dhabi, United Arab Emirates. J. Zoo Wildl. Med. 40(3), 445-452.

Paré, J.A. and Sigler, L. 2016. An overview of reptile fungal pathogens in the genera Nannizziopsis, Paranannizziopsis and Ophidiomyces. J. Herpetol. Med. Surg. 26(1-2), 46-53.

Pasmans, F., Blahak, S., Martel, A. and Pantchev, N. 2008. Introducing reptiles into a captive collection: The role of the veterinarian. Vet. J. 175(1), 53-68.

Rossier, C., Hoby, S., Wenker, C., Brawand, S.G., Thomann, A., Brodard, I., Jermann, T. and Posthaus, H. 2016. Devrieseasis in a plumed basilisk (Basiliscus plumifrons) and chinese water dragons (Physignatus cocincinus) in a zoologic collection. J. Zoo Wildl. Med. 47(1), 280-285.

Schmidt-Ukaj, S., Loncaric, I., Klang, A., Spergser, J., Häbich, A.C. and Knotek, Z. 2014. Infection with Devriesea agamarum and Chrysosporium guarroi in an inland bearded dragon (Pogona vitticeps). Vet. Dermatol. 25(6), 555-558. 